\title{
Seroepidemiology of Toxoplasma gondii infection in patients with liver disease in eastern China
}

\author{
A. L. TIAN ${ }^{1}$, G. X. LI ${ }^{1,2}$, H. M. ELSHEIKHA ${ }^{3}$, D. S. GARDNER ${ }^{3}$,

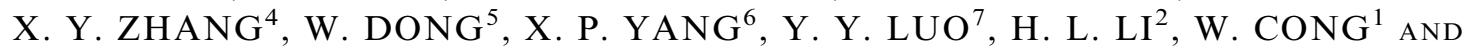 \\ X. Q. ZHU ${ }^{1 *}$ \\ ${ }^{1}$ State Key Laboratory of Veterinary Etiological Biology, Key Laboratory of Veterinary Parasitology of Gansu \\ Province, Lanzhou Veterinary Research Institute, Chinese Academy of Agricultural Sciences, Lanzhou, Gansu \\ Province, People's Republic of China \\ ${ }^{2}$ School of Basic Medicine, Dali University, Dali, Yunnan Province, People's Republic of China \\ ${ }^{3}$ Faculty of Medicine and Health Sciences, School of Veterinary Medicine and Science, University of \\ Nottingham, Sutton Bonington Campus, Loughborough, LE12 5RD, UK \\ ${ }^{4}$ Affiliated Hospital of Medical College, Qingdao University, Qingdao, Shandong Province, People's Republic of \\ China \\ ${ }^{5}$ Weihaiwei People's Hospital, Weihai, Shandong Province, People's Republic of China \\ ${ }^{6}$ Clinical Laboratory, Henan Provincial People's Hospital, Zhengzhou, Henan Province, People's Republic of \\ China \\ ${ }^{7}$ Department of Veterinary Medicine, Southwest University, Rongchang, Chongqing, People's Republic of China
}

Received 6 March 2017; Final revision 30 May 2017; Accepted 6 June 2017;

first published online 5 July 2017

\section{SUMMARY}

The role of the protozoan parasite Toxoplasma gondii in the pathogenesis of liver disease has recently gained much interest. The aim of this study was to determine the prevalence and risk factors associated with $T$. gondii infection in patients with liver disease from three cities in Shandong and Henan provinces, China. A case-control study was conducted from December 2014 to November 2015 and included 1142 patients with liver disease and 1142 healthy controls. Serum samples were collected from all individuals and were examined with enzyme-linked immunosorbent assay for the presence of anti-T. gondii $\operatorname{IgG}$ and IgM antibodies. Information on the demographics, clinical, and lifestyle characteristics of the participants was collected from the medical records and by the use of a questionnaire. The prevalence of anti-T. gondii $\operatorname{IgG}$ was $19 \cdot 7 \%$ in patients with liver disease compared with $12 \cdot 17 \%$ in the controls. Only 13 patients had anti-T. gondii IgM antibodies compared with 12 control individuals $(1 \cdot 14 \%$ vs. $1 \cdot 05 \%$, respectively). The highest seroprevalence was detected in patients with liver cancer $(22 \cdot 13 \%)$, followed by hepatitis patients $(20 \cdot 86 \%)$, liver cirrhosis patients $(20 \cdot 42 \%)$, and steatosis patients $(20 \%)$. Multivariate logistic regression analysis indicated that consumption of raw meat (odds ratio $(\mathrm{OR})=1 \cdot 32 ; 95 \%$ confidence interval $(\mathrm{CI})$ $1 \cdot 01-1 \cdot 71 ; P=0.03)$ and source of drinking water from wells $(\mathrm{OR}=1 \cdot 56 ; 95 \%$ CI $1 \cdot 08-2 \cdot 27 ; P=$ $0 \cdot 01)$ were independent risk factors for $T$. gondii infection in liver disease patients. These findings indicate that $T$. gondii infection is more likely to be present in patients with liver disease. Therefore, efforts should be directed toward health education of populations at high risk of $T$. gondii infection and measures should be taken to protect vulnerable patients with liver disease.

Key words: Case-control study, liver disease, risk factors, seroprevalence, Toxoplasma gondii.

\footnotetext{
* Author for correspondence: X. Q. Zhu, State Key Laboratory of Veterinary Etiological Biology, Lanzhou Veterinary Research Institute, Chinese Academy of Agricultural Sciences, Lanzhou, Gansu Province 730046, People's Republic of China. (Email: xingquanzhu1@hotmail.com)
} 


\section{INTRODUCTION}

Toxoplasmosis is a parasitic disease caused by infection with the intracellular apicomplexan protozoan parasite Toxoplasma gondii. This parasite has a worldwide distribution and is able to infect any warmblooded animal [1]. Infections caused by $T$. gondii continue to impose a significant public health threat because it can cause hydrocephaly, retinochoroiditis, mental retardation, and even death in developing fetus and life-threatening encephalitis in organ transplant recipients, individuals with AIDS, or receiving immunosuppressive therapy [2-4]. T. gondii is one of the most successful protozoan parasites owing to its ability to manipulate the immune system and because of the various means (e.g. waterborne, foodborne, transplacental, or organ transplant) by which the parasite can infect the host and establish a latent infection [5-9].

Although T. gondii possesses neurotropic and ocular affinities, this parasite can also infect other organs, such as liver, spleen, pancreas, heart, and lymph nodes $[10,11]$. Several relatively small studies have detected association between $T$. gondii infection and various hepatic pathologies, such as hepatitis, granuloma, necrosis, hepatomegaly, jaundice, and cirrhosis [1218]. T. gondii infection has also been linked to abnormal liver function tests $[19,20]$. Liver has been shown to be the major site of tissue pathology during acute, lethal toxoplasmosis in mice [21]. An association between the amount of $T$. gondii antigens and increased number of hepatic stellate cells (HSCs) has been reported in the liver of acutely infected mice, suggesting a role for HSCs in the pathogenesis of $T$. gondii-induced hepatitis [22]. T. gondii infection has also been shown to induce several transcriptomic and proteomic changes in the liver of infected mice [23, 24].

In recent years, interest in assessing and understanding the relationship between $T$. gondii infection and liver disease in humans has surged [25, 26], probably due to the huge global burden and public health importance of both $T$. gondii infection [27] and liver diseases [28]. As considerable results exist linking $T$. gondii infection to hepatic damage, and there are no available data about the correlation between liver disease and T. gondii infection in China, it is important to identify any evidence linking $T$. gondii infection to liver disease in humans in China. Therefore, the present study was conducted to investigate the seroprevalence of and risk factors associated with $T$. gondii infection in patients with liver disease from three major cities in Shandong and Henan provinces in eastern China using enzyme-linked immunosorbent assay (ELISA). Our study advances the literature of the association between liver disease and $T$. gondii infection.

\section{MATERIALS AND METHODS}

\section{Ethics statement}

The protocol of the study was reviewed and approved by the Ethics Committee of the Medical College of Qingdao University, Weihaiwei People's Hospital and Henan Provincial People's Hospital. Patients were informed about the aim of the study, and they all provided written consent for participation in the study. Informed consent was also provided by parents or guardians on behalf of all child participants. The control sera were collected with agreement from volunteers.

\section{Study sites}

The study was conducted in two provinces (Shandong and Henan) located in eastern China (Fig. 1). Two cities were selected in Shandong province, namely Qingdao and Weihai. Qingdao is located at the southeastern tip of Shandong province, eastern China ( $35^{\circ}$ $35^{\prime} \mathrm{N}-37^{\circ} 09^{\prime} \mathrm{N}, 119^{\circ} 30^{\prime} \mathrm{E}-121^{\circ} 00^{\prime} \mathrm{E}$ ), and is a key economic center, famous for its cultural heritage and attractive scenery. Weihai is located at the eastern tip of Shandong province $\left(36^{\circ} 41^{\prime} \mathrm{N}-37^{\circ} 35^{\prime} \mathrm{N}, 121^{\circ} 11^{\prime}\right.$ $\left.\mathrm{E}-122^{\circ} 42^{\prime} \mathrm{E}\right)$. The annual average temperature is $12^{\circ} \mathrm{C}$ and the average precipitation is over $800 \mathrm{~mm}$. Zhengzhou $\left(31^{\circ} 23^{\prime} \mathrm{N}-36^{\circ} 22^{\prime} \mathrm{N}, 110^{\circ} 21^{\prime} \mathrm{E}-116^{\circ} 39^{\prime} \mathrm{E}\right)$ is the capital of Henan province, which has a subtropical semi-humid monsoon climate with a hot, humid, and rainy summer; long and cold winter; and an annual average temperature of $14^{\circ} \mathrm{C}$.

\section{Study design and data collection}

A case-control study was designed to identify prevalence and risk factors associated with $T$. gondii infection. The study was conducted from December 2014 to November 2015 and included 1142 inpatients hospitalized for the diagnosis or treatment of liver disease. The clinical cases included patients with various forms of hepatic disease, including liver cirrhosis $(n=333)$, hepatitis $(n=326)$, liver cancer $(n=253)$, steatosis $(n=205)$, and other hepatic disease $(n=25)$. An equivalent number of seronegative control subjects ( $n=1142)$, of similar 


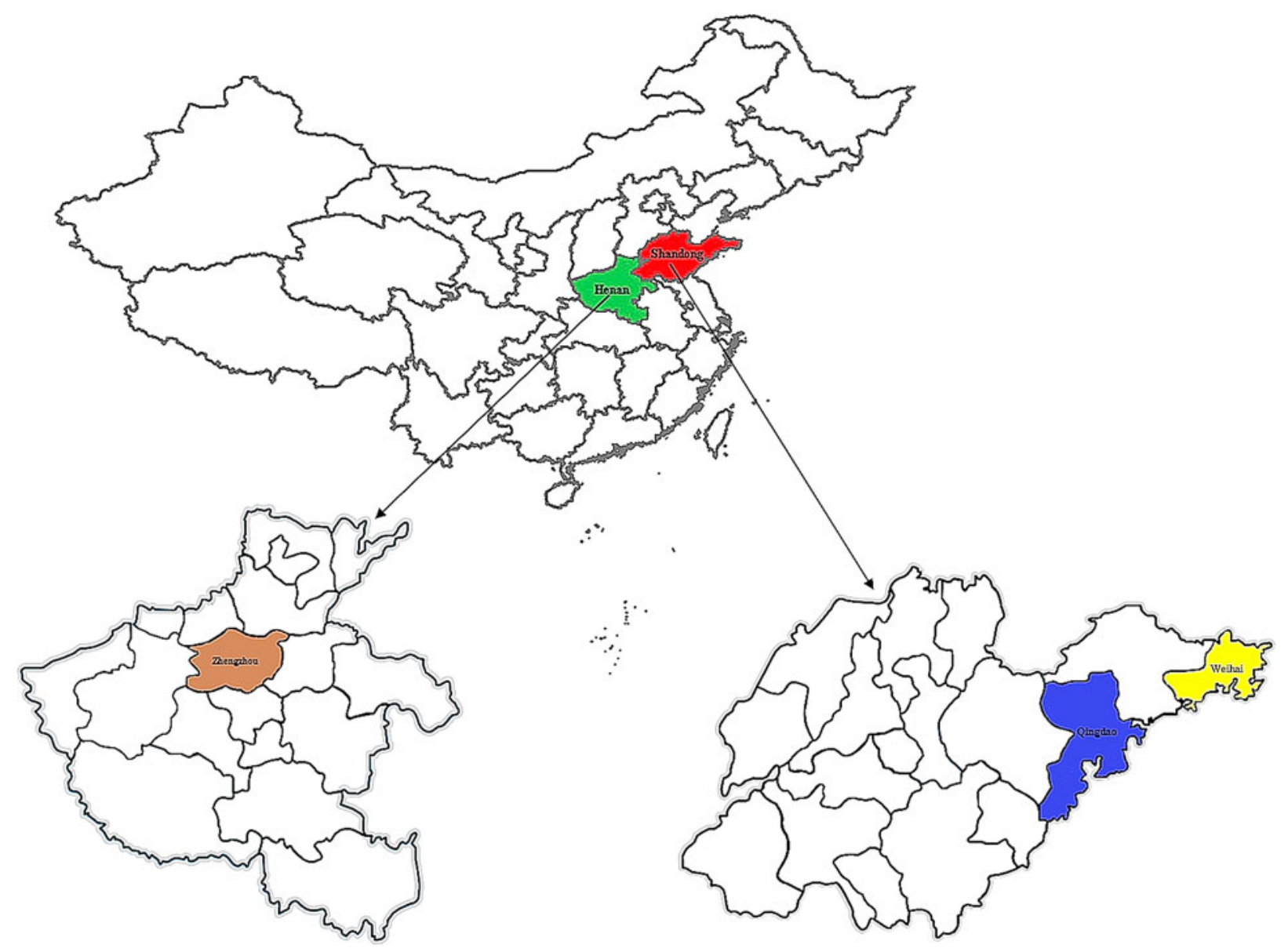

Fig. 1. Location maps of the study sites in eastern China showing the location of Qingdao, Weihai, and Zhengzhou cities targeted in the present prevalence study.

age and gender from each of the three geographic regions were included in the study. Demographics, such as age, gender, and area of residence, were obtained from the computerized inpatient registry of cases or by asking the control participants. A questionnaire was distributed to all participants in order to obtain information about their lifestyle and feeding habits, including the history of close contact with cats or dogs at home (yes/no), consumption of raw vegetables (yes/no), exposure to soil (yes/no), consumption of raw/undercooked meat (yes/ no), and the source of drinking water (none, running water, wells). All personal information was anonymized and treated as strictly confidential.

\section{Serum collection and serological testing}

About $5 \mathrm{ml}$ of venous blood were drawn from each participant. Blood samples were kept overnight at ambient temperature in order to allow clot formation and were centrifuged at $1000 \mathrm{~g}$ for $10 \mathrm{~min}$. The sera were collected in $2 \mathrm{ml}$ Eppendorf tubes and were stored at $4{ }^{\circ} \mathrm{C}$ for $24-72 \mathrm{~h}$ until transportation in an icebox to State Key Laboratory of Veterinary Etiological Biology, Lanzhou Veterinary Research Institute, Chinese Academy of Agricultural Sciences, Gansu Province, where samples were kept at $-20{ }^{\circ} \mathrm{C}$ until analysis. Serum samples were analyzed for the presence of anti- $T$. gondii $\operatorname{IgG}$ and IgM antibodies (using commercially available ELISA kits; Demeditec Diagnostics GmbH, Germany) according to the manufacturer's instructions. Positive and negative serum controls were included in every plate.

\section{Statistical analysis}

Comparison between cases and controls for continuous variables, such as age, used one-way analysis of variance and are reported as mean \pm s.D. For categorical variables, the $\chi^{2}$ test or the Fisher's exact test was used and results are expressed either as percentage of total (number of cases or control) or median (IQR), 
Table 1. Demographics of the study population and seroprevalence of Toxoplasma gondii infection

\begin{tabular}{|c|c|c|c|c|c|c|c|}
\hline \multirow[b]{2}{*}{ Variable } & \multicolumn{3}{|c|}{ Cases with liver diseases $(n=1142)$} & \multicolumn{3}{|c|}{ Control group $(n=1142)$} & \multirow[b]{2}{*}{$P$-value } \\
\hline & No. tested & No. positive & Prevalence $(\%)$ & No. tested & No. positive & Prevalence $(\%)$ & \\
\hline \multicolumn{7}{|c|}{ Age group (years) } & \multirow[t]{7}{*}{$0 \cdot 10$} \\
\hline 30 or less & 103 & 23 & $22 \cdot 33$ & 254 & 40 & $15 \cdot 75$ & \\
\hline $31-40$ & 153 & 29 & 18.95 & 169 & 16 & $9 \cdot 47$ & \\
\hline $41-50$ & 309 & 74 & 23.95 & 297 & 38 & $12 \cdot 79$ & \\
\hline $51-60$ & 243 & 38 & $15 \cdot 64$ & 150 & 13 & $8 \cdot 67$ & \\
\hline $61-70$ & 190 & 36 & 18.95 & 132 & 17 & $12 \cdot 88$ & \\
\hline$>70$ & 144 & 33 & $22 \cdot 92$ & 140 & 26 & $18 \cdot 57$ & \\
\hline \multicolumn{7}{|l|}{ Gender } & \multirow[t]{3}{*}{$0 \cdot 008$} \\
\hline Male & 595 & 114 & $19 \cdot 16$ & 532 & 67 & 12.59 & \\
\hline Female & 547 & 119 & $21 \cdot 76$ & 610 & 83 & $13 \cdot 61$ & \\
\hline \multicolumn{7}{|c|}{ Geographic region } & \multirow{4}{*}{$<0.001$} \\
\hline Qingdao & 395 & 87 & $22 \cdot 03$ & 382 & 44 & $11 \cdot 52$ & \\
\hline Weihai & 349 & 95 & $27 \cdot 22$ & 494 & 67 & $13 \cdot 56$ & \\
\hline Zhengzhou & 398 & 51 & $12 \cdot 81$ & 266 & 39 & $14 \cdot 66$ & \\
\hline \multicolumn{7}{|c|}{ Residence area } & \multirow[t]{3}{*}{$0 \cdot 71$} \\
\hline Urban & 532 & 108 & $20 \cdot 30$ & 649 & 66 & $10 \cdot 17$ & \\
\hline Rural & 610 & 125 & $20 \cdot 49$ & 493 & 84 & $17 \cdot 04$ & \\
\hline
\end{tabular}

as appropriate. To identify factors associated with T. gondii $\mathrm{IgG}$ seropositivity, a univariate approach was first used with a cut-off of $\leqslant 0 \cdot 10$ to identify contributing factors to include in a multivariate analysis. Logistic regression was then used to identify significantly contributing factors with all variables included from univariate analyses. Only variables significant at $P<0.05$ were retained in multivariate analysis. The statistical model included age and gender (fitted first, to account for minor variation in the datasets; adjustment used the Mantel-Haenszel test). All statistical analyses were performed using Genstat v18 (VSNi, Rothampsted, UK). Odds ratios (ORs) with 95\% confidence intervals (CIs) were calculated to indicate effect size of predictive variables. A $P$-value $<0.05$ was considered to be statistically significant.

\section{RESULTS}

T. gondii $\mathrm{IgG}$ antibodies were detected in $225(19 \cdot 7 \%)$ of patients with liver disease, which was significantly higher $(P<0 \cdot 001)$ than the level detected in the control individuals $(139 ; 12 \cdot 17 \%)$. In contrast, $T$. gondii IgM antibodies were only detected in 13 patients and 12 controls $(1 \cdot 14 \%$ vs. $1 \cdot 05 \%$, respectively, $P=$ $0 \cdot 84)$. Table 1 describes the seroprevalance according to patients' age, gender, geographic region, and residence area. The highest seroprevalence of $T$. gondii infection was found in patients with liver disease whose ages ranged from 41 to 50 (Table 1). The seroprevalence of $T$. gondii infection among patients with liver disease from rural areas $(20 \cdot 49 \%) v s$. urban areas $(20 \cdot 30 \%)$ was not significantly different $(P=0 \cdot 71)$.

Association of $T$. gondii infection with clinical liver disease is shown in Table 2. Patients with liver disease had a higher frequency of $T$. gondii infection per se, with those patients with a diagnosis of liver cancer $>$ cirrhosis $>$ hepatitis having greatest seropositivity. Multivariate analysis revealed that consumption of raw meat $(\mathrm{OR}=1.32 ; 95 \%$ CI $1.01-1 \cdot 71 ; P=0.03)$ and source of drinking water from wells $(\mathrm{OR}=1 \cdot 56$; 95\% CI $1.08-2.27 ; P=0.01$ ) were the only independent risk factors for $T$. gondii infection in liver disease patients (Table 3). Other variables did not show any significant association with $T$. gondii infection.

\section{DISCUSSION}

The present work revealed a higher prevalence of anti- $T$. gondii $\operatorname{IgG}$ antibodies in patients with liver disease compared with the control subjects. It is possible that the immune-compromised status associated with liver disease might make the patients become more vulnerable to infection with $T$. gondii compared with healthy individuals $[29,30]$. One previous study did not detect an association between $T$. gondii infection and liver disease in patients from Mexico [26]. However, the prevalence rate obtained in our study was lower than that reported in Egypt in patients 
Table 2. The seroprevalence of Toxoplasma gondii in patients with various hepatic disorders in China

\begin{tabular}{|c|c|c|c|c|}
\hline \multirow[b]{2}{*}{ Clinical disease } & \multicolumn{4}{|c|}{ Patients with anti- $T$. gondii IgG antibodies } \\
\hline & No. tested & No. positive $(\%)$ & OR $(95 \% \mathrm{CI})$ & $P$-value \\
\hline None & 1003 & $139(13 \cdot 86)$ & Reference & - \\
\hline Liver cirrhosis & 267 & $66(24 \cdot 72)$ & $2 \cdot 05(1 \cdot 46-2 \cdot 86)$ & $<0.001$ \\
\hline Hepatitis & 259 & $67(25 \cdot 87)$ & $1 \cdot 85(1 \cdot 34-2 \cdot 56)$ & $<0.001$ \\
\hline Liver cancer & 199 & $54(27 \cdot 14)$ & $2 \cdot 48(1.69-3.65)$ & $<0.001$ \\
\hline Steatosis & 167 & $38(22 \cdot 75)$ & $1 \cdot 59(1 \cdot 06-3 \cdot 39)$ & $0 \cdot 02$ \\
\hline Other $^{1}$ & 25 & $0(0)$ & - & - \\
\hline Total & 1920 & $364(18 \cdot 96)$ & $1 \cdot 91(1 \cdot 51-2 \cdot 42)$ & $<0.001$ \\
\hline
\end{tabular}

OR, odds ratio; CI, confidence interval.

${ }^{1}$ Include amoebic liver abscess, hepatic hemangioma, hepatic cyst, focal liver lesions, and hepatocellular liver disease.

Table 3. Multivariate analysis of risk factors for Toxoplasma gondii infection in patients with liver disease

\begin{tabular}{|c|c|c|c|c|c|c|c|}
\hline \multirow[b]{2}{*}{ Variable } & \multirow{2}{*}{$\begin{array}{l}\text { Number } \\
\text { tested }\end{array}$} & \multirow{2}{*}{$\begin{array}{l}\text { Number } \\
\text { positive }\end{array}$} & \multirow{2}{*}{$\begin{array}{l}\text { Prevalence } \\
(\%)\end{array}$} & \multicolumn{2}{|c|}{ Univariate analysis ${ }^{1}$} & \multicolumn{2}{|c|}{ Multivariate analysis $^{2}$} \\
\hline & & & & OR $(95 \% \mathrm{CI})^{*}$ & $P$-value & OR $(95 \% \mathrm{CI})$ & $P$-value \\
\hline Cats at home & 744 & 35 & $4 \cdot 7$ & $1 \cdot 08(0 \cdot 77-1 \cdot 51)$ & $0 \cdot 61$ & - & - \\
\hline Dogs at home & 744 & 29 & $3 \cdot 9$ & $1 \cdot 13(0 \cdot 78-1 \cdot 63)$ & $0 \cdot 49$ & - & - \\
\hline Consumption of raw vegetables & 744 & 73 & $9 \cdot 81$ & $0 \cdot 90(0 \cdot 69-1 \cdot 18)$ & $0 \cdot 48$ & - & - \\
\hline Exposure to soil & 744 & 97 & $13 \cdot 04$ & $1 \cdot 13(0 \cdot 87-1 \cdot 46)$ & $0 \cdot 34$ & - & - \\
\hline $\begin{array}{l}\text { Consumption of raw/undercooked } \\
\text { meat }\end{array}$ & 744 & 81 & $10 \cdot 89$ & $1 \cdot 30(1 \cdot 00-1 \cdot 69)$ & $0 \cdot 04$ & $1 \cdot 32(1 \cdot 01-1 \cdot 71)$ & $0 \cdot 03$ \\
\hline Source of drinking water: none & 398 & 48 & $12 \cdot 06$ & Reference & - & - & - \\
\hline Running water & 587 & 135 & $23 \cdot 0$ & $1 \cdot 27(0 \cdot 95-1 \cdot 68)$ & $0 \cdot 09$ & $1 \cdot 09(0 \cdot 79-1 \cdot 50)$ & $0 \cdot 56$ \\
\hline Wells & 157 & 42 & $26 \cdot 75$ & $1 \cdot 79(1 \cdot 26-2 \cdot 54)$ & $<0 \cdot 001$ & $1 \cdot 56(1 \cdot 08-2 \cdot 27)$ & $0 \cdot 01$ \\
\hline
\end{tabular}

OR, odds ratio; CI, confidence interval.

${ }^{1}$ Statistical model tested individual effect, after correction for age, gender, and geographic region.

${ }^{2}$ Statistical model excluded factors not significant on univariate analysis, but included consumption of raw/undercooked meat and source of drinking water from wells, after correction for age, gender, and geographic region.

with hepatic disease $65 \cdot 5 \%$ vs. $27 \%$ in the controls [31], in late-stage cirrhotic patients $92.6 \%$ vs. $15 \%$ controls, and chronic HCV non-cirrhotic patients $76.9 \%$ vs. $40 \%$ controls [32]. Another study in patients with chronic liver disease form Egypt reported 30\% vs. $6 \%$ in controls [25]. Differences in prevalence rates among these studies could be attributed to geographic variability, heterogeneity of the studied populations, or in the diagnostic approaches employed. In our study, the result of $T$. gondii IgM antibodies $(1 \cdot 14 \%$ vs. $1 \cdot 05 \%$, respectively, $P=0.84$ ) was not significantly different between cases and control. This finding is consistent with the results reported in patients with liver disease in Egypt, where no significant difference $(P=0.610)$ in the level of anti- $T$. gondii $\operatorname{IgM}$ antibodies was detected in late-stage $(13.6 \%)$ and earlystage $(12 \cdot 8 \%)$ patients compared with $7 \cdot 5 \%$ controls [32].
Recent transcriptomic [23] and proteomic [24] findings showed that $T$. gondii infection can dysregulate several signaling pathways, especially immune response pathway in the liver of infected mice. Acute $T$. gondii can also reduce the activity of hepatic enzyme butyrylcholinesterase, which plays a role in immune response and lipid synthesis [33]. Additionally, infection with $T$. gondii $\mathrm{RH}$ strain can elicit strong inflammatory response and high levels of $\mathrm{T}$ helper cell type 1 cytokines, which damage the liver [21]. Therefore, it is sensible to assume that the pathogenesis of liver damage during $T$. gondii infection can be induced by parasite-derived factors that disrupt host signaling pathways and/or host-mediated factors (and other as yet unknown mechanisms).

Further, we found that patients with liver cancer had the highest $T$. gondii seroprevalence compared with other forms of liver diseases. This is expected 
because patients treated with antineoplastic agents are more likely to be infected with $T$. gondii $[34,35]$. The immune suppression associated with chemotherapeutic treatment of patients with malignancies can result in the reactivation of latent $T$. gondii infection [36]. An association between $T$. gondii seropositivity and consumption of sheep meat in patients with live disease has been reported in Mexico $(\mathrm{OR}=8.69 ; 95 \%$ CI $1.02-73.71 ; P=0 \cdot 04)$ [26]. However, in our study, none of the lifestyle variables, including contact with cats or dogs at home, consumption of raw vegetables, consumption of raw/undercooked meat, or exposure to soil, was found to have a significant association with $T$. gondii seropositivity. Interestingly, multivariate analysis revealed only two independent risk factors for $T$. gondii infection, which were the consumption of raw meat $(\mathrm{OR}=1.32 ; 95 \% \mathrm{CI} 1.01-1 \cdot 71 ; P=0.03)$ and source of drinking water from wells $(\mathrm{OR}=1.56$; 95\% CI $1 \cdot 08-2 \cdot 27 ; P=0 \cdot 01)$.

In China, cats are popular companion animals, but the public are not well informed of the risk of environmental contamination with $T$. gondii oocysts [37]. Humans can be infected with $T$. gondii oocysts through drinking water contaminated with cat feces [38], which has been identified as a public health threat [39]. Felids are the only definitive hosts for T. gondii, and primary infection results in shedding of millions of environmentally resistant non-sporulated oocysts within approximately 2 weeks. Oocysts become infectious within 1-5 days, depending on the climatic conditions [40]. The identification of the source of drinking water as one of the two independent risk factors for $T$. gondii infection in our study necessitates that measures should be taken to protect the public in general and immunocompromised individuals in particular from the risk of water-borne infection with $T$. gondii oocysts. Likewise, the identification of consumption of raw meat as a significant risk factor for $T$. gondii infection confirms the overwhelming evidence regarding the epidemiological relevance of meat-borne infection with $T$. gondii [2, 38, 41, 42]. There is a clear need for increased education of health professionals and the public about the potential transmission of $T$. gondii via drinking water from wells or consumption of raw meat in order to reduce the risk and burden of toxoplasmosis in China.

\section{CONCLUSION}

To our knowledge, this is the largest study that specifically investigates the epidemiology of $T$. gondii infection in association with liver disease. Additionally, our study examined patients with various clinical forms of liver disease and utilized multivariate analysis to determine which factors are independently associated with the risk of $T$. gondii infection in patients with liver disease. Our results underscore the need for monitoring the presence of $T$. gondii infection in patients with liver disease, especially those with immunocompromised status. Efficient lifestyle and environmental intervention strategies are also needed in order to reduce the risk of water-borne and meatborne transmission of $T$. gondii oocysts. Additional studies are needed to define which $T$. gondii genotype is most likely to be associated with hepatopathy in China.

\section{ACKNOWLEDGEMENTS}

Project support was provided by Fundamental Research Funds of the Chinese Academy of Agricultural Sciences (Grant No. Y2016JC05) and the Agricultural Science and Technology Innovation Program (ASTIP) (Grant No. CAAS-ASTIP-2014LVRI-03).

\section{DECLARATION OF INTEREST}

None.

\section{REFERENCES}

1. Elmore SA, et al. Toxoplasma gondii: epidemiology, feline clinical aspects, and prevention. Trends in Parasitology 2010; 26: 190-196.

2. Elsheikha HM. Congenital toxoplasmosis: priorities for further health promotion action. Public Health 2008; 122: $335-353$.

3. Ong E. Common AIDS-associated opportunistic infections. Clinical Medicine 2008; 8: 539-543.

4. Weiss LM, Dubey JP. Toxoplasmosis: a history of clinical observations. International Journal for Parasitology 2009; 39: 895-901.

5. Tenter AM, et al. Toxoplasma gondii: from animals to humans. International Journal for Parasitology 2000; 30: $1217-1258$.

6. Dawson D. Foodborne protozoan parasites. International Journal of Food Microbiology 2005; 103: 207-227.

7. Jones JL, Dubey JP. Foodborne toxoplasmosis. Clinical Infectious Diseases 2012; 55: 845-851.

8. Villena I, et al. Evaluation of a strategy for Toxoplasma gondii oocyst detection in water. Applied and Environmental Microbiology 2004; 70: 4035-4039.

9. Patel R. Disseminated toxoplasmosis after liver transplantation. Clinical Infectious Diseases 1999; 29: 705-706. 
10. Walker M, et al. Parasitic central nervous system infections in immunocompromised hosts. Clinical Infectious Diseases 2005; 40: 1005-1015.

11. Balasundaram MB, et al. Outbreak of acquired ocular toxoplasmosis involving 248 patients. Archives of Ophthalmology 2010; 128: 28-32.

12. Weitberg $\mathbf{A B}$, et al. Acute granulomatous hepatitis in the course of acquired toxoplasmosis. New England Journal of Medicine 1979; 300: 1093-1096.

13. Karasawa T, et al. Localized hepatic necrosis related to cytomegalovirus and Toxoplasma gondii. Acta Pathologica Japonica 1981; 31: 527-534.

14. Tiwari I, et al. Cholestatic jaundice due to toxoplasma hepatitis. Postgraduate Medical Journal 1982; 58: 299-300.

15. Hassan MM, et al. Parasitic causes of hepatomegaly in children. Journal of the Egyptian Society of Parasitology 1996; 26: 177-189.

16. Ustun S, et al. Incidence of toxoplasmosis in patients with cirrhosis. World Journal of Gastroenterology 2004; 10: 452-454.

17. Doğan N, et al. Toxoplasmic hepatitis in an immunocompetent patient. Türkiye Parazitoloji Dergisi 2007; 31: 260-263.

18. Shapira Y, et al. Serum markers of infections in patients with primary biliary cirrhosis: evidence of infection burden. Experimental and Molecular Pathology 2012; 93: 386-390.

19. Ortego TJ, et al. Toxoplasmic chorioretinitis and hepatic granulomas. American Journal of Gastroenterology 1990; 85: 1418-1420.

20. Wendum D, et al. Fatal disseminated toxoplasmosis in a toxoplasma seropositive liver transplant recipient. Journal of Clinical Pathology 2002; 55: 637.

21. Mordue DG, et al. Acute toxoplasmosis leads to lethal overproduction of Th1 cytokines. Journal of Immunology 2001; 167: 4574-4584.

22. Atmaca HT, et al. Hepatic stellate cells increase in Toxoplasma gondii infection in mice. Parasites and Vectors 2013; 6: 135.

23. He JJ, et al. Transcriptomic analysis of mouse liver reveals a potential hepato-enteric pathogenic mechanism in acute Toxoplasma gondii infection. Parasites and Vectors 2016; 9: 427.

24. He JJ, et al. Proteomic profiling of mouse liver following acute Toxoplasma gondii infection. PLoS ONE 2016; 11: e0152022.

25. El-Sayed NM, et al. Toxoplasma gondii infection and chronic liver diseases: evidence of an association. Tropical Medicine and Infectious Diseases 2016; 1: 7.

26. Alvarado-Esquivel C, et al. Toxoplasma gondii infection and liver disease: a case-control study in a northern Mexican population. Parasites and Vectors 2011; 4: 75.
27. Montoya JG, Liesenfeld O. Toxoplasmosis. Lancet 2004; 363: 1965-1976.

28. Mokdad AA, et al. Liver cirrhosis mortality in 187 countries between 1980 and 2010: a systematic analysis. BMC Medicine 2014; 12: 145.

29. Bonacini M, et al. Duodenal and hepatic toxoplasmosis in a patient with HIV infection: review of the literature. American Journal of Gastroenterology 1996; 91: 1838-1840.

30. Mastroianni A, et al. Liver toxoplasmosis and acquired immunodeficiency syndrome. Recenti Progressi in Medicina 1996; 87: 353-355.

31. Ghanam ME, et al. Evaluation of the role of some parasitic infections as a cause of acute and chronic hepatic diseases. Journal of the Egyptian Society of Parasitology 2001; 31: 37-42.

32. El-Nahas HA, et al. Toxoplasma gondii infection among chronic hepatitis C patients: a case-control study. Asian Pacific Journal of Tropical Medicine 2014; 7: 589-593.

33. Da Silva AS, et al. Relationship between butyrylcholinesterase activity and liver injury in mice acute infected with Toxoplasma gondii. Pathology, Research and Practice 2013; 209: 95-98.

34. Cong W, et al. Toxoplasma gondii infection in cancer patients: prevalence, risk factors, genotypes and association with clinical diagnosis. Cancer Letters 2015; 359: 307-313.

35. Yazar S, et al. Investigation of anti-Toxoplasma gondii antibodies in patients with neoplasia. Journal of Medical Microbiology 2004; 53: 1183-1186.

36. Evering T, Weiss LM. The immunology of parasite infections in immunocompromised hosts. Parasite Immunology 2006; 28: 549-565.

37. Du F, et al. Survey on the contamination of Toxoplasma gondii oocysts in the soil of public parks of Wuhan, China. Veterinary Parasitology 2012; 184: 141-146.

38. Jones JL, et al. Risk factors for Toxoplasma gondii infection in the United States. Clinical Infectious Diseases 2009; 49: 878-884.

39. Torrey EF, Yolken RH. Toxoplasma oocysts as a public health problem. Trends in Parasitology 2013; 29: 380-384.

40. Jones JL, Dubey JP. Waterborne toxoplasmosis recent developments. Experimental Parasitology 2010; 124: 10-25.

41. Elsheikha HM, et al. Seroprevalence of and risk factors for Toxoplasma gondii antibodies among asymptomatic blood donors in Egypt. Parasitology Research 2009; 104: 1471-1476.

42. Wilking $\mathbf{H}$, et al. Prevalence, incidence estimations, and risk factors of Toxoplasma gondii infection in Germany: a representative, cross-sectional, serological study. Scientific Reports 2016; 6: 22551. 